\title{
Preventing Capacity Drop at Isolated Merging Bottleneck through Variable Speed Limit Control
}

\author{
Junhyung Lee and Jaekang Lee* \\ Korea Institute of Civil Engineering and Building Technology, \\ 283, Goyangdae-ro, Ilsanseo-gu, Goyang-si, Gyeonggi-do 10223, Korea
}

(Received August 28, 2019; accepted October 7, 2019)

Keywords: variable speed limit, merging bottleneck, density control, throughput

The traffic flow control strategy is crucial to properly discharge a queue at a bottleneck. In particular, breakdown prevention is a key objective to maintain high-throughput traffic. Many researchers have studied variable speed limit (VSL) algorithms for their own purposes. However, previous VSL studies have mainly focused on traffic safety aspects, and fluctuation mechanisms depending on VSL algorithms have not been fully investigated. In this study, we designed a VSL algorithm to adjust the density (or occupancy) to the optimal range to increase the throughput of the bottleneck. Demand-capacity analysis and simulation results indicate that flow reduction is achieved through VSL. VSL temporarily mitigates input flows by delaying the downstream arrival time. In addition, the results confirm that VSL can effectively control the traffic density to properly handle the congestion of a given merging bottleneck. According to previous research studies and empirical data analysis, there is a relationship between critical density (occupancy) and breakdown. The VSL algorithm clearly shows that throughput at a merging bottleneck can increase through density control. This mechanism sheds some light on traffic control using VSL, which could be adopted in the foreseeable future.

\section{Introduction}

The throughput at a bottleneck section can be maximized near a critical density. However, when the density of the roadway section exceeds a critical value, the discharge flow rate is much lower than the capacity. ${ }^{(1-3)}$ This phenomenon is well known as a capacity drop. Cassidy and Bertini ${ }^{(4)}$ asserted that queue formation is accompanied by a relatively low discharge rate. Yuan et al $^{(5)}$ and $\mathrm{Oh}$ and $\mathrm{Yeo}^{(6)}$ defined the capacity drop as the difference between the queue discharge rate and the pre-queue capacity. The oblique coordination system $(O \text {-curve })^{(4,7-9)}$ is commonly used to visually identify flow changes at each measurement location. ${ }^{(6,10-12)}$

The main purpose of this study is to prevent the capacity drop at an isolated merging bottleneck by adjusting the density of the bottleneck section with variable speed limit (VSL) control. To adjust the density of the bottleneck section, the arrival flow rate of the bottleneck should be decreased by reducing the speed of approaching vehicles. This is the basic concept

${ }^{*}$ Corresponding author: e-mail: jaekang.lee@kict.re.kr https://doi.org/10.18494/SAM.2019.2567 
of this VSL algorithm. Lin et al. ${ }^{(13)}$ already reduced the queue length and maximized the throughput using VSL control in highway work zones.

There are some similar cases that maximized the throughput with density (or occupancy) control. Cassidy and Rudjanakanoknad ${ }^{(9)}$ increased the discharge rate at a merging bottleneck through ramp metering. This metering strategy mitigated the shoulder lane queue, and high outflow is restored. According to Cassidy and Rudjanakanoknad, ${ }^{(9)}$ a capacity drop occurs when the occupancy reaches $27 \%$, and the shoulder lane queue begins to shrink when the occupancy drops below $22 \%$. This result implies that the throughput at the bottleneck can be optimally maintained within the occupancy range from 22 to $27 \%$. Chung et al. ${ }^{(14)}$ also studied the relationship between vehicle density and discharge flow. Their findings indicated that the density clearly coincided with capacity drops. At the bottleneck, there was a decrease in discharge rate once the queue formed, while the discharge rate was entirely recovered when the density dropped below the critical density. These results indicate that capacity drops can be prevented by controlling the density (or occupancy) of the bottleneck section.

In summary, the discharge flow at the bottleneck is mainly dependent on the density or occupancy of the bottleneck, and the density or occupancy can be controlled by adjusting the speed of approaching vehicles from the upstream bottleneck.

\section{Empirical Analysis}

\subsection{Observation of the capacity drop at an isolated merging bottleneck}

Our study focuses on increasing the throughput at an isolated merging bottleneck. As a first step, the empirical relationship between a capacity drop and the occupancy at an isolated merging bottleneck is confirmed. The merging bottleneck studied here is located on the eastbound Hobeop junction of the No. 50 Expressway in Korea. The Hobeop junction is an interchange of the No. 35 Expressway and No. 50 Expressway. It has 3-4 main lanes in one direction and frequently experiences congestion on weekends due to leisure travelers. The Hobeop junction has loop detectors at $1.1 \mathrm{~km}$ intervals. The study data were collected on Saturday, May 14, 2016. Each loop detector obtained volume, speed, and occupancy data every $30 \mathrm{~s}$ (Fig. 1).

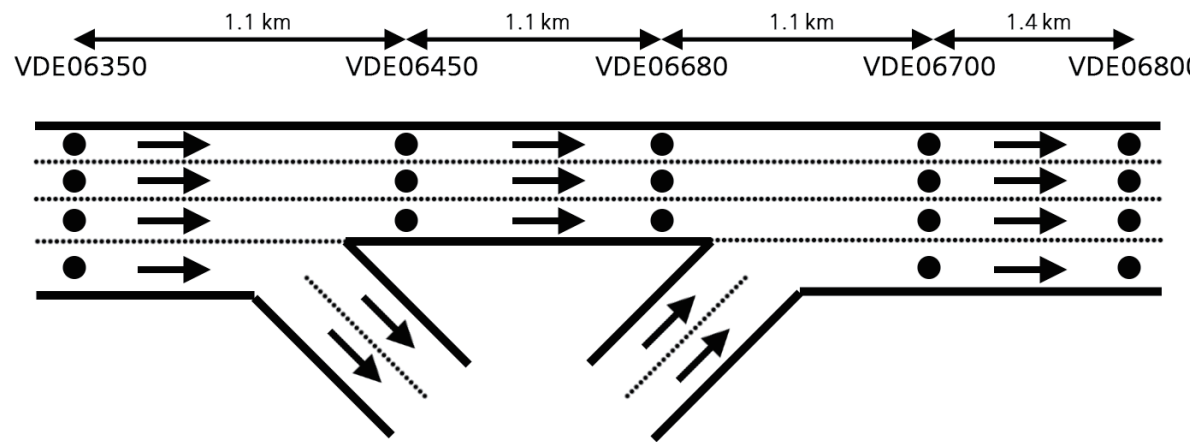

Fig. 1. Road geometry of Hobeop junction. 
Figure 2 shows the curve of the cumulative vehicle counts measured at three locations (VDE06450, VDE06680, and VDE06700). The $O$-curve in Fig. 2 was drawn using Eq. (1). ${ }^{(4,7-9)}$ In Fig. 2, the throughputs of VDE06680 and VDE06450 are reduced at 6:14 a.m. and 6:25 a.m., respectively. Congestion occurs at the bottleneck (VDE06680) at 6:14 a.m. and spills back to the upstream (VDE06450) at 6:25 a.m. The downstream of the bottleneck (VDE06700) has not been congested during the same time period.

$$
O(t)=V(t)-q_{0} \times\left(t-t_{0}\right)
$$

where

$O(t)=$ orthogonal quantity of the queue at time $t$ (veh),

$V(t)=$ cumulative virtual vehicle count to time $t$ (veh),

$q_{0}=$ background reduction (veh/h), and

$t_{0}=$ curve start time $(\mathrm{h})$.

\subsection{Observation of the throughput}

The throughput of the merging bottleneck was observed at VDE06680. ${ }^{(10)}$ The data obtained from lanes 1 and 2 were considered representative of all lanes. Figure 3 shows the $O$-curve at the bottleneck (VDE06680). When the discharge flow becomes low at 6:14 a.m., the occupancy increases up to $30 \%$, whereas the downstream remains in the free-flow state. This phenomenon is observed in both lanes. Thus, there is a significant relationship between the occupancy and the throughput as shown by Cassidy and Rudjanakanoknad. ${ }^{(9)}$

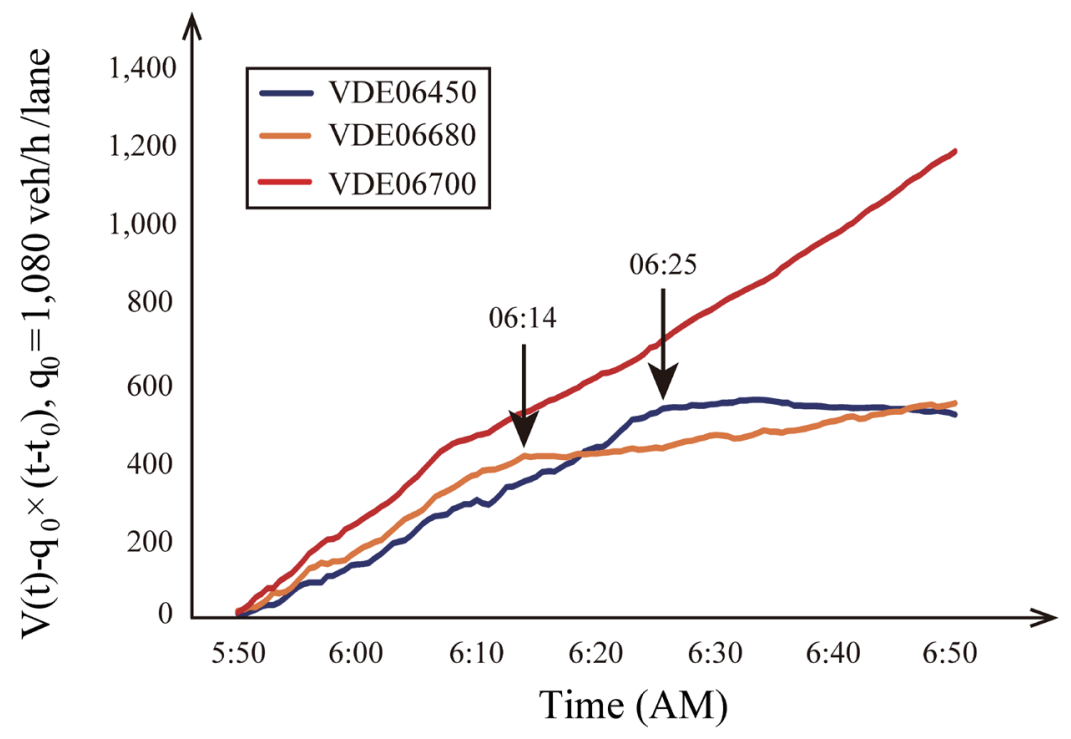

Fig. 2. (Color online) $O$-curves at three locations. 

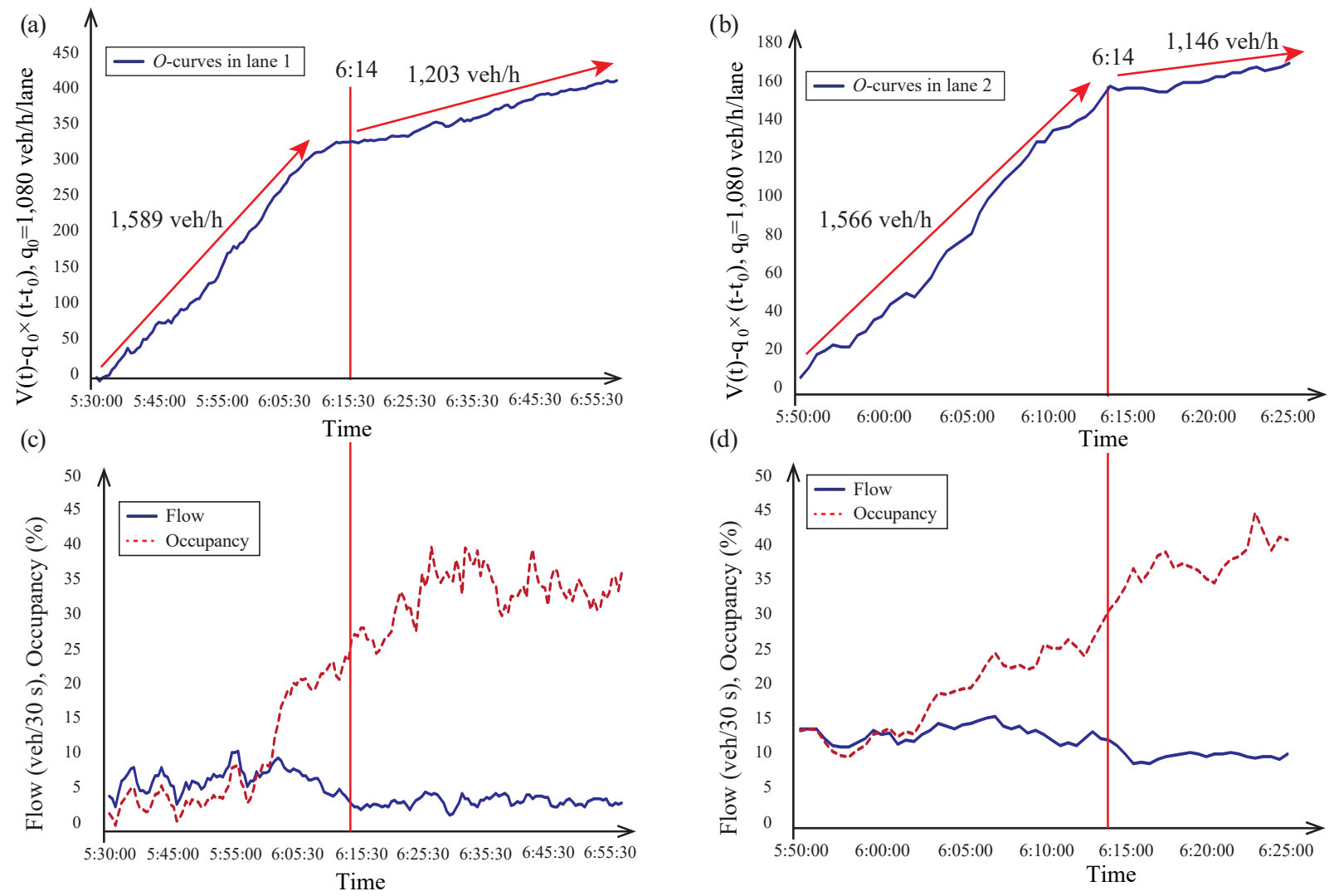

Fig. 3. (Color online) VDE06680 bottleneck: (a) $O$-curves in lane 1, (b) $O$-curves in lane 2, (c) $150 \mathrm{~s}$ movingaverage flow and occupancy in lane 1, and (d) 150 s moving-average flow and occupancy in lane 2.

Once the occupancy of the bottleneck exceeds a critical value, drivers cannot maintain sufficient headway for cruise driving. Thus, the flow transitions into a stop-and-go state and the throughput decreases. ${ }^{(15)}$ According to previous studies, the throughput would recover once the occupancy remained sufficiently low..$^{(9,14)}$ Therefore, the occupancy should be maintained within the optimal range between the critical occupancy and the threshold occupancy at which breakdown occurs. For example, from the results of empirical data analysis, the occupancy of the Hobeop junction area should be maintained within 18.16 and $30.67 \%$ to achieve a maximum traffic flow efficiency.

\subsection{Transition of the traffic condition}

According to the empirical analysis of the Hobeop junction area, the management of occupancy (or density) is a key mechanism in maintaining the maximum throughput at the bottleneck. Occupancy management significantly correlates with breakdown to the congested state. However, the transition mechanisms when activating the bottleneck and recovering from it are significantly different. Owing to these two different transitions, the bottleneck is considered a two-capacity concept. ${ }^{(2)}$ Thus, we observe the transition mechanism using a timetracing flow-occupancy diagram (Fig. 4). 


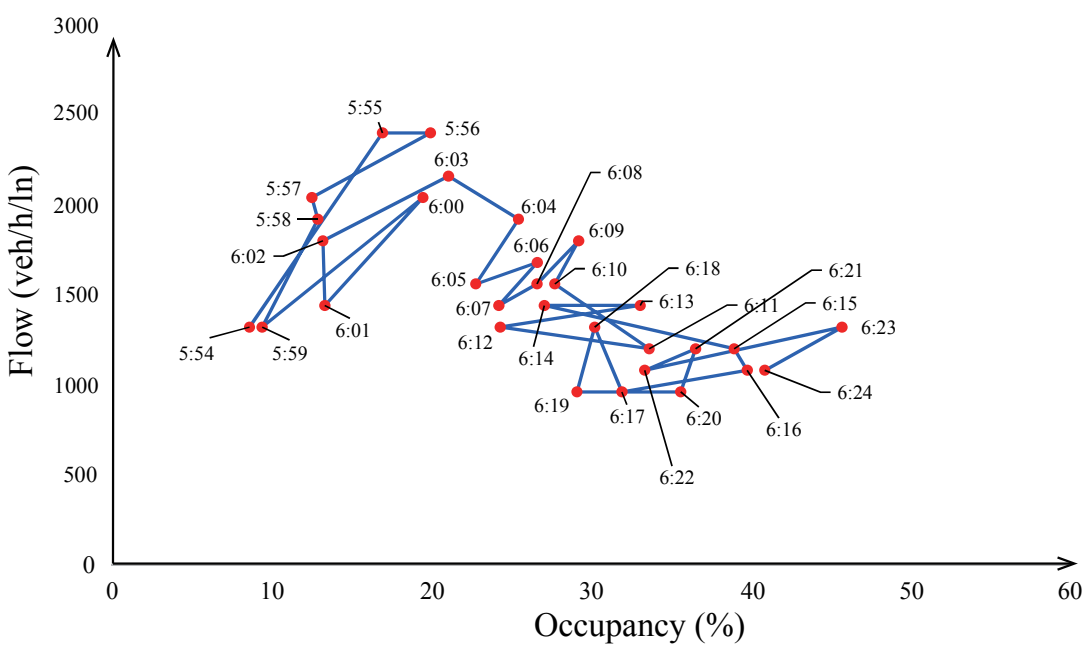

(a)

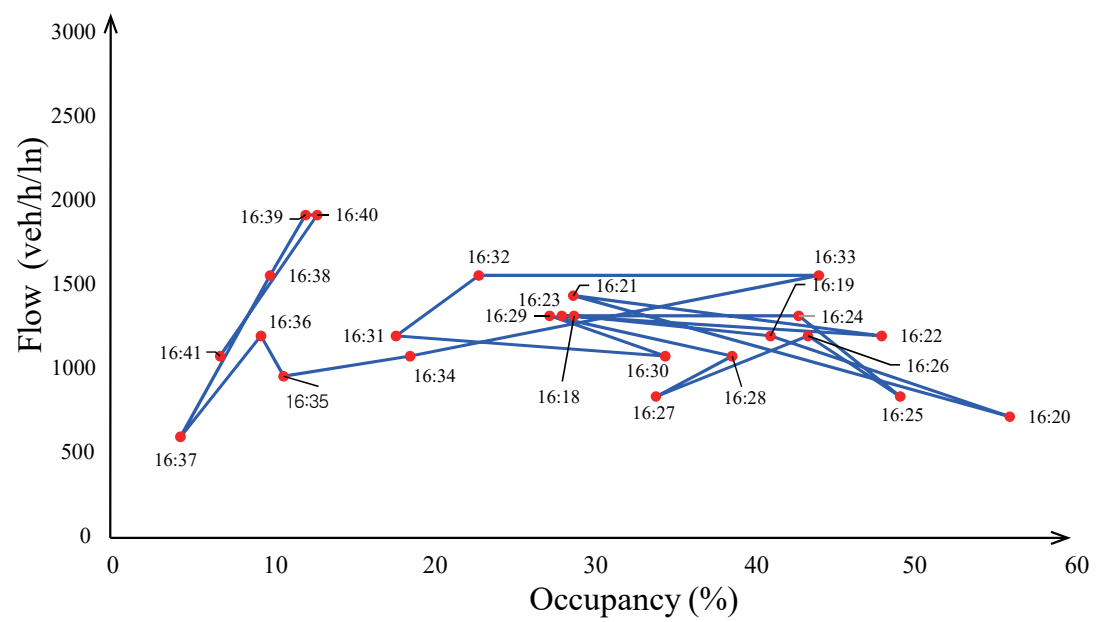

(b)

Fig. 4. (Color online) Time-tracing transition mechanism. (a) Transition from free-flow to congestion and (b) transition from congestion to free-flow.

When the traffic transitions to a congested state, the occupancy varies near the capacity [Fig. 4(a)]. Conversely, when recovering from congestion, the traffic data cannot reach the capacity level and directly move to the free-flow state [Fig. 4(b)]. The throughput does not increase until the traffic returns to the free-flow state. Thus, when the occupancy varies near the capacity, breakdown can be prevented by keeping the occupancy below the critical value. The following VSL algorithm focuses on this perspective and tries to adjust the density to the target value. There is a reason why the proposed VSL algorithm adjusts the density and not the occupancy. The density is generally used in theoretical work, and empirical studies have used the occupancy because it is widely measured by loop detectors. The occupancy correlates well with the density and can typically replace it owing to the difficulty in measuring the density. ${ }^{(16)}$ For the theoretical background of the proposed VSL algorithm, we adopt the density as a target object, but it can simply be replaced by the occupancy depending on the field condition. 


\section{VSL Control Algorithm}

This VSL algorithm is used to adjust the density to the optimal range of values to prevent breakdown. The VSL control process is shown in Fig. 5. The VSL control speed should be sufficient and reasonable. If the VSL control speed is lower than the critical speed, the unsaturated upstream drivers will not follow the VSL control.

First, we collect the traffic data and compute the critical density at the control area. Next, we analyze the current densities of the control and upstream areas. From the observed current density, we forecast the next time-step density. The next time-step density can be forecast on the basis of the expected arrival and departure flow rates. When the operator expects that the next time-step density would exceed the critical value, we compute the next time-step VSL control speed. In contrast, if the next time-step density is lower than the critical value, we return to the free-flow speed or remain in the current state.

One of the most effective ways to control the density is adjusting the upstream input flow. ${ }^{(13)}$ This strategy can control the arrival flow rate to the bottleneck and affect the density. Thus, to reduce the density, the arrival flow rate must be lower than the current departure flow rate.

In the queuing theory, the downstream arrival curve has a free-flow travel time gap with the upstream departure curve [Fig. 6(a)]. When we assume that all vehicles immediately follow the VSL control, the downstream arrival time is delayed on the basis of each vehicle's location. The slope of the arrival curve at the bottleneck is decreased during the time $t_{\text {adj }}$ [Fig. 6(a)] because of the different delayed times based on the location when the vehicles slow down. The time $t_{\text {adj }}$ is the travel time estimated under the VSL control speed [Eq. (2)]. After the VSL is fully applied, the slope of the arrival curve returns to the original arrival flow rate.

$$
t_{a d j}=\left(\frac{l}{V_{a d j}}\right),
$$

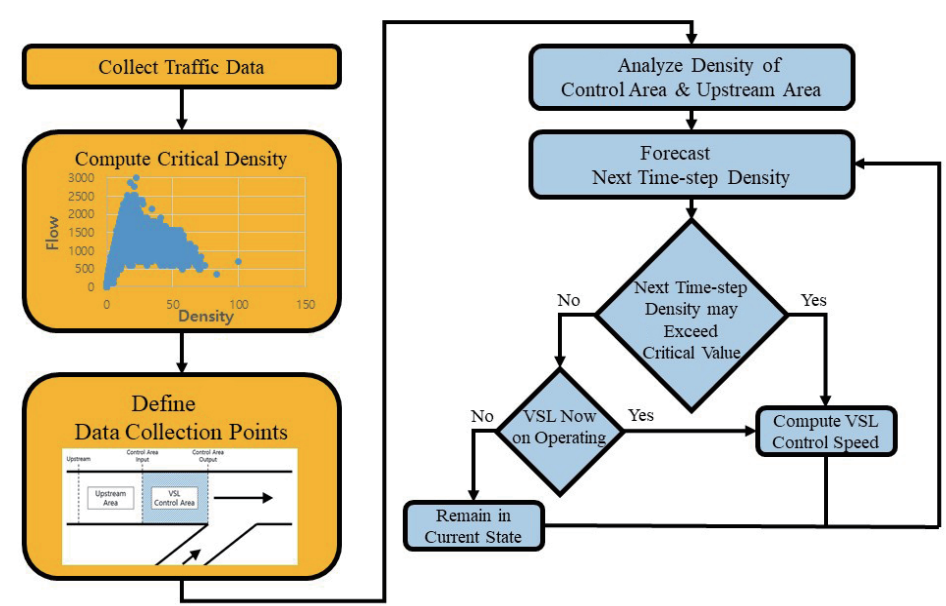

Fig. 5. (Color online) VSL control process. 


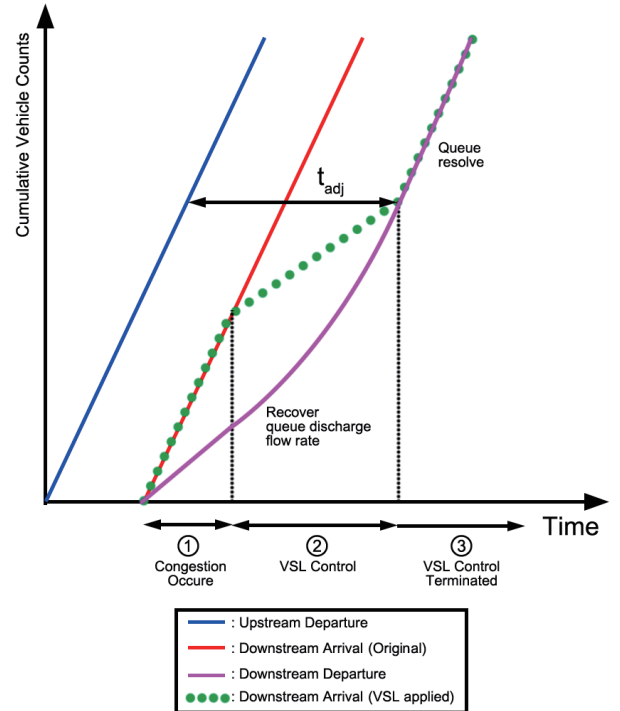

(a)

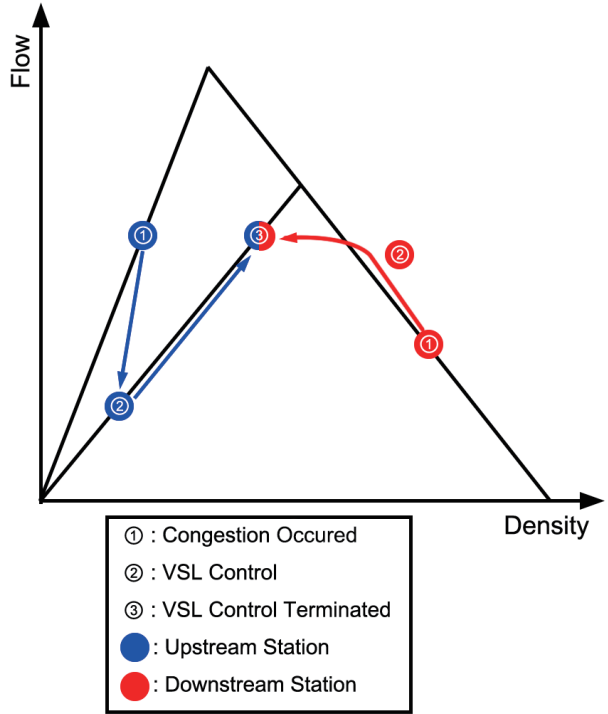

(b)

Fig. 6. (Color) (a) Cumulative arrival-departure curve when VSL is applied. (b) Flow-density diagram when VSL is applied.

where

$t_{a d j}=$ estimated travel time from upstream to downstream under the VSL control (h),

$V_{a d j}=$ adjusted VSL speed of approaching vehicles $(\mathrm{kph})$, and

$l=$ length of the VSL control area $(\mathrm{km})$.

Considering that the downstream arrival time is delayed during the time $t_{a d j}$, the arrival flow rate at the bottleneck is adjusted on the basis of Eq. (3). If the arrival flow rate decreases to a value below the discharge rate, the density at the VSL control area consequently decreases. To adjust the density to the optimal range, the VSL speed should be computed using Eq. (4). The breakdown of varying traffic can be prevented by adjusting the density. The critical density (critical occupancy) is determined on the basis of the precollected traffic data of that site.

$$
q_{a d j}=\frac{q_{0} \times \frac{l}{V_{f}}}{t_{a d j}}=\frac{V_{a d j}}{V_{f}} q_{0},
$$

where

$q_{a d j}=$ adjusted arrival flow rate $(\mathrm{veh} / \mathrm{h})$,

$q_{0}=$ arrival flow rate $(\mathrm{veh} / \mathrm{h})$, and

$V_{f}=$ free-flow speed $(\mathrm{kph})$.

$$
\left(D_{0}-D_{\text {breakdown }}\right) \times l \geq\left(q_{0}-q_{a d j}\right) \times t_{a d j} \geq\left(D_{0}-D_{\text {critical }}\right) \times l,
$$




$$
\left(D_{0}-D_{\text {breakdown }}\right) \times l \geq q_{0} \times\left(\frac{V_{f}-V_{a d j}}{V_{f}}\right) \times \frac{l}{V_{a d j}} \geq\left(D_{0}-D_{\text {critical }}\right) \times l,
$$

where

$D_{0}=$ current density $(\mathrm{veh} / \mathrm{km})$,

$D_{\text {breakdown }}=$ breakdown occurrence density $(\mathrm{veh} / \mathrm{km})$, and

$D_{\text {critical }}=$ critical density $(\mathrm{veh} / \mathrm{km})$.

The density at the bottleneck is adjusted via VSL control using Eq. (4). However, the arrival traffic flow recovers to the original traffic demand flow rate after the time $t_{a d j}$ has passed. The operator can recurrently adjust the speed limit on the basis of the stair stepping cycle if the density in the control area is not effectively transformed to the optimal range. In Fig. 6, we trace (1)-

(3) states on the basis of the demand-capacity analysis and flow-density diagram.

(1) The queue forms at the bottleneck, but the upstream still remains in the free-flow state.

(2) After starting the VSL control, the downstream arrival time and arrival flow rate decreases. The density of the upstream control area decreases consequently. The downstream departure curve gradually increases.

(3) Upstream demand returns to the original rate. However, the density at the bottleneck is adjusted under the critical value. The queue at the bottleneck finally diminishes.

\section{VSL Algorithm Simulation}

To verify the VSL algorithm, we designed a simple Verkehr In Städten - SIMulation modell (VISSIM) simulation network (Fig. 7). VISSIM is one of the well-known microscopic traffic simulation programs. The simulation network is designed similarly to the Hobeop junction area. The Hobeop junction consists of three main lines and two on-ramp lanes. The locations of data collection points are also the same as those of the Hobeop junction study site.

First, we input the vehicles into the simulation network, which has a free-flow speed of $100 \mathrm{kph}(62.1 \mathrm{mph})$, and collect the traffic data. The approaching traffic demand is observed $7 \mathrm{~km}(4.3 \mathrm{mi})$ upstream to suppress the effect of the spill-back queue. The number of downstream arriving vehicles is determined $200 \mathrm{~m}(656.2 \mathrm{ft})$ upstream from the bottleneck.

In the simulation network, the reduced-speed areas are set every $50 \mathrm{~m}(164.0 \mathrm{ft})$ in a $5 \mathrm{~km}$ VSL control area. Basic simulation tests indicated that the arrival time at the bottleneck

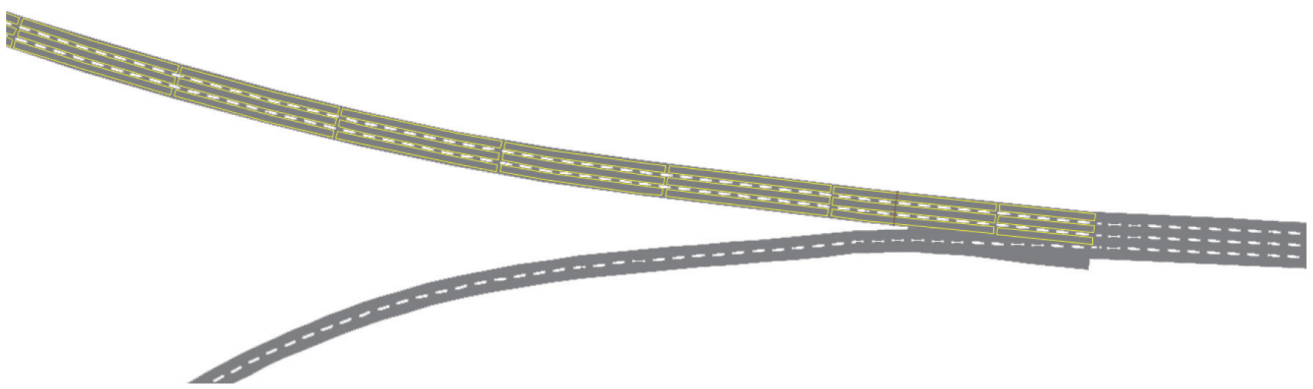

Fig. 7. (Color online) VISSIM simulation network. 
is delayed by $150 \mathrm{~s}$ when limiting the speed by $50 \mathrm{kph}(31.1 \mathrm{mph})$ from $1380 \mathrm{~s}$ (Fig. 8). Additionally, the arrival flow reduction is observed from 1380 to $1680 \mathrm{~s}$. These results are nearly identical using Eqs. (2) and (3). According to both equations, the results should display a $180 \mathrm{~s}$ delay during $1380-1740 \mathrm{~s}$. These results illustrate that the VSL can effectively reduce the arrival flow rate. The minor error between the theoretical analysis and simulation results might be due to the length of the reduced-speed area. The simulation limits the vehicles' speed every $50 \mathrm{~m}(164.0 \mathrm{ft})$.

As the second step, we examined the ability of the VSL algorithm to control density. As shown in Fig. 9, the critical density of the simulation site is about $100 \mathrm{veh} / \mathrm{km}$. Under non-VSL algorithm conditions, the density exceeds the critical value. However, when conducting the VSL algorithm according to Eq. (4), the density decreases under the critical value. The transition of the new $\mathrm{q}-\mathrm{k}$ diagram shows that the $\mathrm{q}-\mathrm{k}$ data points moved to the free-flow condition. This proposed VSL algorithm would prevent breakdown and increase the throughput.

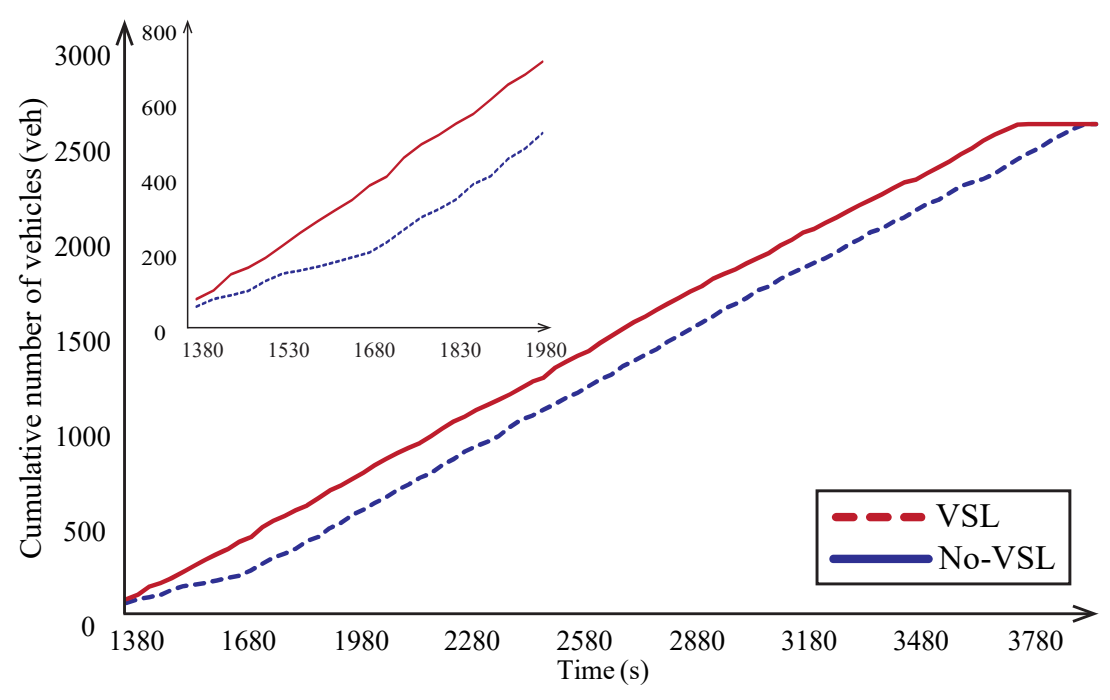

Fig. 8. (Color online) Cumulative number of vehicles in the VISSIM simulation network.

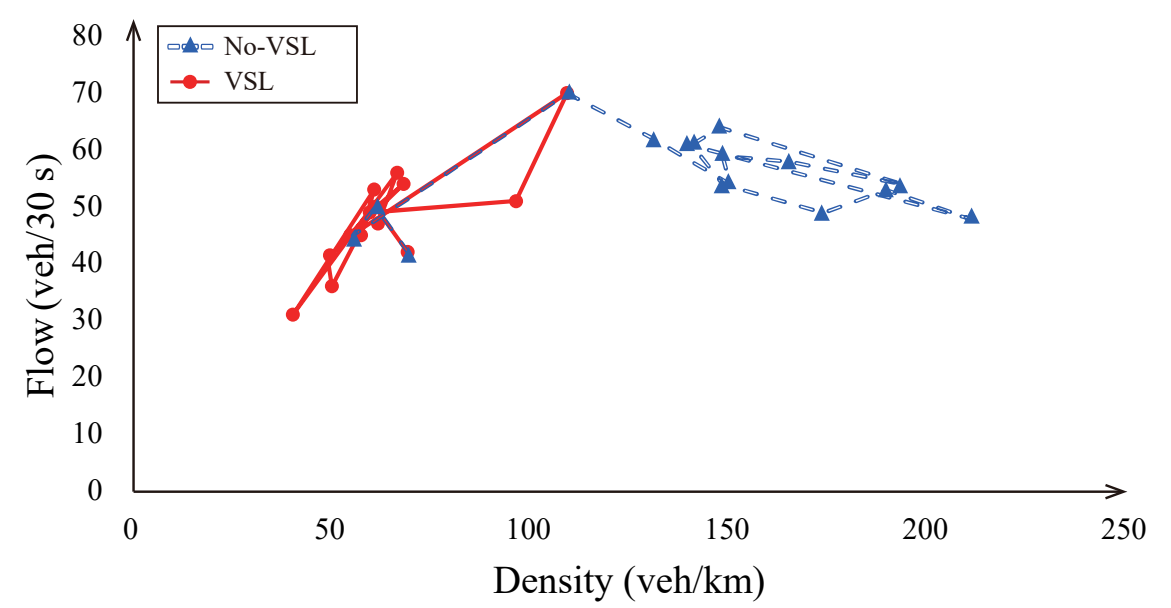

Fig. 9. (Color online) Diagram illustrating a comparative study on the flow-density transition. 


\section{Conclusion}

In this study, we designed a VSL algorithm that can maintain a free-flow state by controlling the density. High densities cause a breakdown of traffic flow and the throughput consequently decreases. According to previous studies ${ }^{(5,9,14)}$ and empirical data analysis, there is a clear relationship between the optimal density (occupancy) range and breakdown. As shown in this study, breakdown occurs when the density (occupancy) exceeds a critical value. Also, the density (occupancy) varies within a specific range prior to the traffic flow becoming unstable. Thus, the core concept of this VSL algorithm is adjusting the density (occupancy) under the critical value to prevent breakdown.

To adjust the density (occupancy) at the bottleneck, the algorithm mitigates the input flow from the upstream. Consequently, the downstream arrival time is delayed and the arrival flow rate is reduced [Fig. 6(a)] by VSL control.

Our simulation results support this theoretical analysis (Fig. 8). The density was sufficiently reduced to a value below the critical value (Fig. 9). These simulation results were significant, as they proved that breakdown was prevented. Traffic flow remained at a stable state with the control of the density at the bottleneck. In other words, this mechanism improved the efficiency of traffic flow, allowing for increased throughput.

Density control through this VSL algorithm leads to improved traffic efficiency at an isolated merging bottleneck within any range of increase in total travel time. However, a long VSL control area extends the effective control time $t_{a d j}$ and helps in the proper adjustment of density (occupancy). This VSL algorithm successfully adjusts the merging bottleneck's throughput through the density control. This mechanism can be applied in real VSL control in the foreseeable future. For advanced VSL control, we need to develop further the next timestep density forecasting technique. An accurate density forecasting technique encourages more effective density control by preventing overcritical density situations.

\section{Acknowledgments}

This work is supported by the Korea Agency for Infrastructure Technology Advancement (KAIA) grant funded by the Ministry of Land, Infrastructure and Transport (Grant 19SCIP-C146569-02).

\section{References}

1 V. F. Hurdle and P. K. Datta: Transp. Res. Rec. 905 (1983) 127. http://onlinepubs.trb.org/Onlinepubs/ trr/1983/905/905-021.pdf

2 J. H. Banks: Transp. Res. Rec. 1320 (1991) 234. http://onlinepubs.trb.org/Onlinepubs/trr/1991/1320/1320-027. pdf

3 F. L. Hall and K. Agyemang-Duah: Transp. Res. Rec. 1320 (1991) 91. http://onlinepubs.trb.org/Onlinepubs/ trr/1991/1320/1320-012.pdf

4 M. J. Cassidy and R. L. Bertini: Transp. Res. Part B: Methodol. 33 (1999) 25. https://doi.org/10.1016/S01912615(98)00023-X

5 K. Yuan, V. L. Knoop, and S. P. Hoogendoorn: Transp. Res. Rec. 2491 (2015) 72. https://doi.org/10.3141/249108 
6 S. Oh and H. Yeo: Transp. Res. Rec. 2286 (2012) 111. https://doi.org/10.3141/2286-13

7 G. F. Newell: Transp. Res. Part B: Methodol. 27 (1993) 281. https://doi.org/10.1016/0191-2615(93)90038-C

8 G. F. Newell: Transp. Res. Part B: Methodol. 27 (1993) 289. https://doi.org/10.1016/0191-2615(93)90039-D

9 M. J. Cassidy and J. Rudjanakanoknad: Transp. Res. Part B: Methodol. 39 (2005) 896. https://doi.org/10.1016/ j.trb.2004.12.001

10 M. J. Cassidy and J. R. Windover: Transp. Res. Rec. 1484 (1995) 73. http://bertini.eng.usf.edu/pdf/cassidy_ windover.pdf

11 A. Srivastava and N. Geroliminis: Transp. Res. Part C: Emerging Technol. 30 (2013) 161. https://doi. org/10.1016/j.trc.2013.02.006

12 S. Oh: Master's Thesis, An Empirical Study on Capacity Drop Phenomenon in Highway Merging Sections (Korea Advanced Instituted of Science and Technology, Daejeon, 2011) .

13 P. W. Lin, K. P. Kang, and G. L. Chang: J. Intell. Transp. Syst. 8 (2004) 155. https://doi. org/10.1080/15472450490492851

14 K. Chung, J. Rudjanakanoknad, and M. J. Cassidy: Transp. Res. Part B: Methodol. 41 (2007) 82. https://doi. org/10.1016/j.trb.2006.02.011

15 S. Oh and H. Yeo: Transp. Res. Part B: Methodol. 77 (2015) 88. https://doi.org/10.1016/j.trb.2015.03.017

16 R. P. Roess, E. S. Prassas, and W. R. McShane: Traffic Engineering (Prentice Hall, N.J, 2011) 4th ed.

\section{About the Authors}

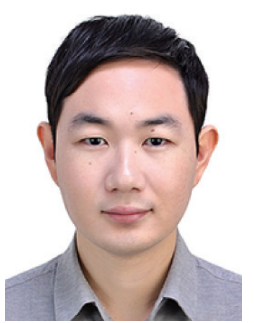

Junhyung Lee is a postdoctoral researcher at the Korea Institute of Civil Engineering and Building Technology. He received his B.E. and Ph.D. degrees from Yonsei University, Korea, in 2013 and 2019, respectively. His research interests are in traffic flow theory, uninterrupted traffic flow, merging bottleneck, and autonomous vehicle driving algorithm.

(takion0424@yonsei.ac.kr)

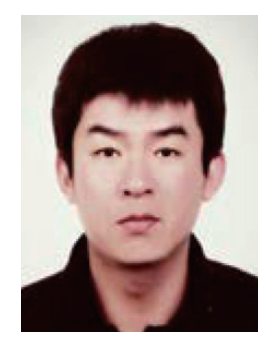

Jaekang Lee is a researcher at the Korea Institute of Civil Engineering and Building Technology. He received his Ph.D. degree from the University of Nottingham, UK, in 2016. His research interests are in GNSS positioning, UAV photogrammetry, and construction automation. (jaekang.lee@kict.re.kr) 